\title{
CASE REPORT: TONGUE GANGRENE AND OROPHARYNGEAL CARCINOMA
}

\author{
Fábio Muradás Girardi', Eduardo Lange Rech², Victória Teles França ${ }^{2}$, \\ Luiz Inácio Roman²
}

\begin{abstract}
Clin Biomed Res. 2016;36(3):168-171
1 Department of Head and Neck Surgery, Hospital Ana Nery. Santa Cruz do Sul, RS, Brazil.
\end{abstract}

2 Medical School, Universidade de Santa Cruz do Sul (UNISC). Santa Cruz do Sul, RS, Brazil.

Corresponding author: Fábio Muradás Girardi fabiomgirardi@gmail.com Department of Head and Neck Surgery, Hospital Ana Nery

Rua Pereira da Cunha, 209 96835-090, Santa Cruz do Sul, RS, Brazil.

\begin{abstract}
Gangrene of the tongue is a condition rarely described in the literature. It generally occurs in association with temporal arteritis and other vasculitides. We described a rare case of tongue necrosis associated with oropharyngeal carcinoma. A 67-male patient, previously submitted to exclusive radiotherapy for a squamous cell carcinoma of the tonsillar region, was admitted to our service with high dysphagia. Computed tomography showed circumferential swelling of the oropharynx, with areas of diffuse contrast uptake and significant reduction of the vascular flow at this level, especially venous return. About 6 months after the onset of symptoms, he was submitted to an urgent tracheostomy for airway obstruction. Then, about 1 month after tracheotomy, the neck skin became ecchymotic, congested, and he started with profuse oropharyngeal bleeding and congestion and thrombosis signs in the tongue. To our knowledge, this entity has not been yet described in the literature.
\end{abstract}

\section{Keywords: Oropharyngeal neoplasms; tongue; thrombosis}

The tongue has an excellent blood supply. The obstruction of any of the lingual arteries hardly causes ischemia because of the rich vascular communications along the base and tip of the tongue, as well as the collateral vessels from pharyngeal and palatine branches ${ }^{1,2}$. Gangrene of the tongue is usually caused by an extensive and bilateral vascular lesion. Temporal arteritis and other vasculitides have been described as the main causes of tongue gangrene. We described an unusual case of tongue thrombosis associated with history of oropharyngeal carcinoma. We did not find another description of this association in the literature.

\section{CASE REPORT}

A 67-male patient of the public health system, ex-smoker and ex-alcoholic, submitted to exclusive radiotherapy for a squamous cell carcinoma of the tonsillar region 14 years ago, was admitted to our service with high dysphagia, progressing for about 1 month. On clinical examination, despite the radiotherapy abnormalities related to the prior treatment, no other findings were present on oral and neck examination. Computed tomography (CT) showed irregularities in the contour of the mucosa in the left side of oropharynx, with an image compatible with lymph node with central necrosis in the retropharyngeal space (figure 1A). A circumferential swelling of the oropharynx, with areas of diffuse contrast uptake and significant reduction of the vascular flow at the oropharyngeal level was present, especially venous return (figure 1B). Flow in the carotid artery and in the internal jugular vein was recovered at the level of the arytenoids (figure 1C). A laryngoscopy was performed under sedation. Despite a diffuse hardening of the oropharynx, we observed no mucosal ulcerations or any focal area of malignancy suspicion. The patient was submitted to multiple pharyngeal biopsies in four different opportunities during the following two months, all of them negative for cancer. We did not have a Positron Emission Tomography (PET-CT) at our disposal in our service. After 3 months of the onset of symptoms, the patient was receiving exclusive nasoenteric tube feeding because of severe dysphagia. About 6 months after 
the onset of symptoms, he was submitted to an urgent tracheotomy for airway obstruction. And about 1 month after tracheostomy the neck skin became ecchymotic (figure 2A), congested, and he started with profuse oropharyngeal bleeding and tongue signs of congestion and thrombosis. The tongue had an ecchymotic and swollen appearance, with purple and grayish discoloration areas, suggesting ischemic process by thrombosis (figure 2B). In two months, the patient died because of pulmonary infectious complications.

\section{DISCUSSION}

Temporal arteritis has been described as one of the main causes of tongue necrosis ${ }^{2-4}$. Polyarteritis nodosa and Systemic Lupus Erythematosus have also been associated with similar cases ${ }^{5,6}$. Storm et al. reported a tongue thrombosis case in a 72-year-old patient with giant cell arteritis ${ }^{7}$. Lekovic et al. ${ }^{8}$ reported the case of a 28-year-old old woman with tongue thrombosis associated with antiphospholipid-antibody syndrome. In the last both cases there was a complete resolution with conservative therapy (corticosteroids

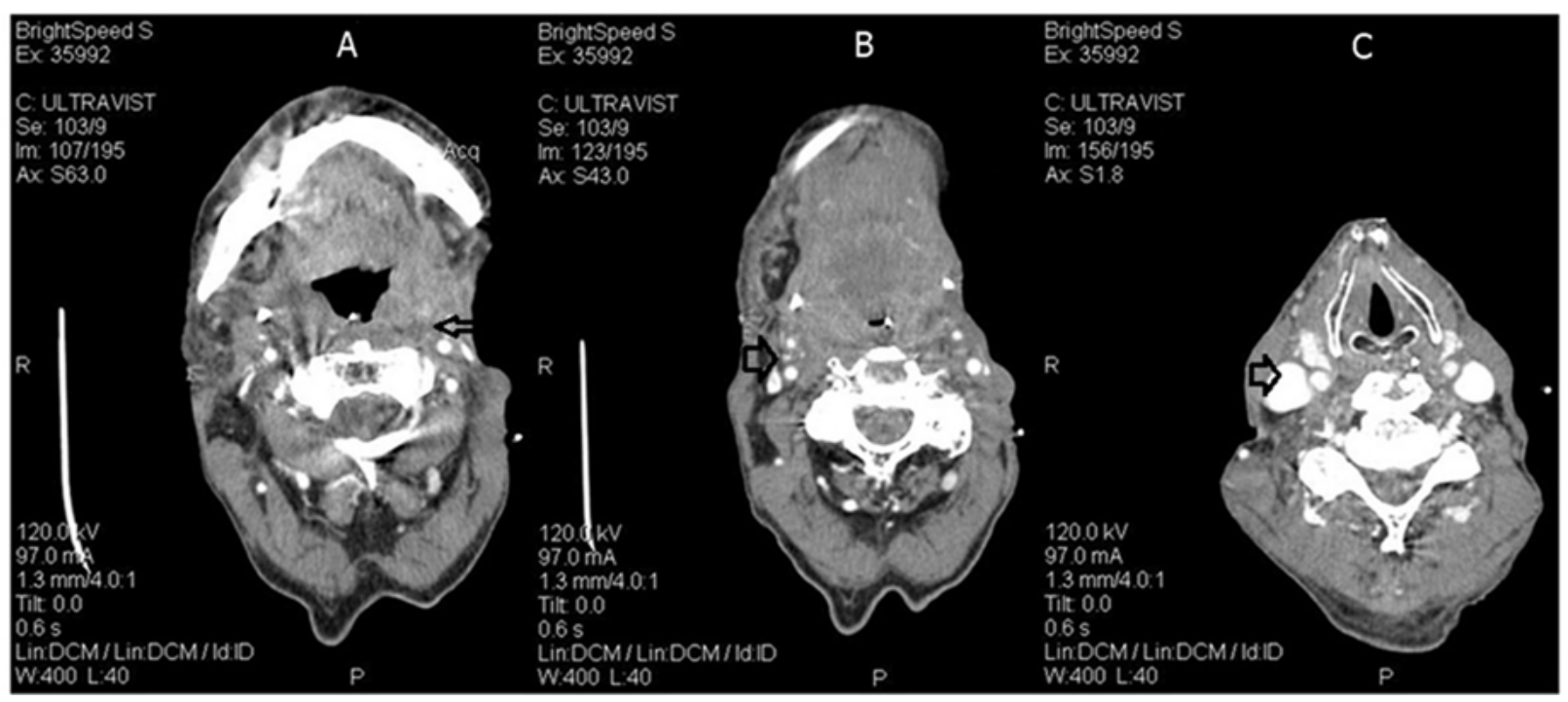

Figure 1: Computed tomography (CT): On the left side of retropharyngeal space, there is a lymph node with central necrosis (arrow, figure A). A circumferential swelling of the oropharynx, with areas of diffuse contrast uptake and significant reduction of the vascular flow at the oropharyngeal level is present (arrow, figure B). Flow in the carotid artery and in the internal jugular vein was recovered at the level of the arytenoids (arrow, figure C).

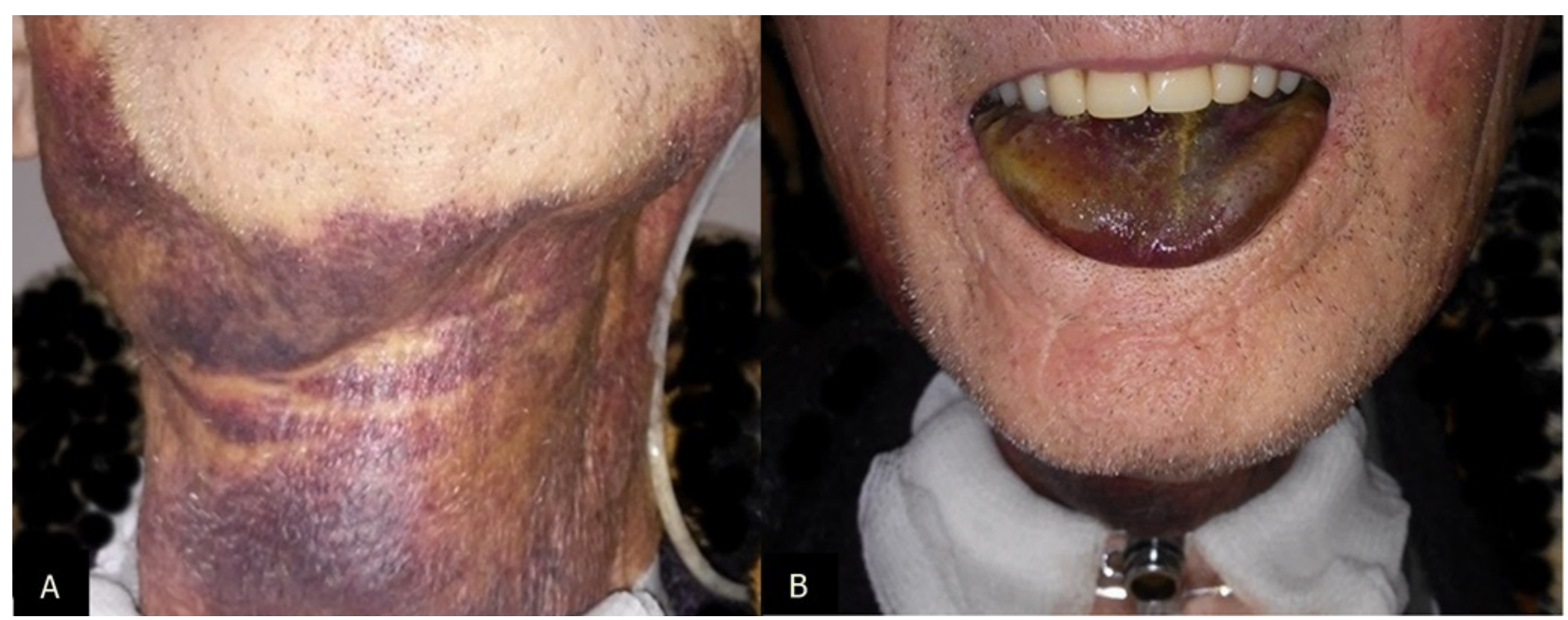

Figure 2: A: The neck 1 month after tracheostomy. Note that the neck skin became ecchymotic and congested; B: The tongue 1 month after tracheostomy. Note the ecchymotic and swollen appearance, with purple and grayish discoloration areas. 
and anticoagulants, respectively), without debridement necessity.

Buch et al. ${ }^{9}$ described a tongue necrosis case associated with the use of epirubicin and cyclophosphamide in a 62-year-old woman with breast cancer. As in our case, the patient developed rapid swelling of the tongue and airway obstruction, requiring an urgent tracheostomy. Encabo et al. described the case of a 64-year-old patient with tongue necrosis after adjuvant radiotherapy for supraglottic laryngeal carcinoma primarily treated with laser resection ${ }^{1}$. The authors believe that a bilateral arterial thrombosis on a pre-existing atherosclerotic territory was responsible for the tongue necrosis.

Similar cases associated with systemic use of vasopressors, bowel infarction, and transient ischemic attack have also been described ${ }^{2}$. Kamatani et al. ${ }^{2}$ described the case of an 82-year-old man with tongue necrosis secondary to disseminated intravascular coagulation post-hepatectomy for hepatocellular carcinoma. Morris et al. ${ }^{10}$ reported a case of tongue gangrene after a cardiogenic shock in a 79-year-old patient.

We did not find any reports of tongue thrombosis or gangrene associated with oral or oropharyngeal cancer. We believe tongue gangrene in this case probably occurred due to a reduction of the vascular flow, especially venous return, aggravating the pharyngeal edema and compromising the viability of tongue tissue. Oral phlebothrombosis is extremely rare. A group from Bauru, state of São Paulo, described a case of a localized tongue phlebothrombosis ${ }^{11}$. The lesion was about $1.5 \mathrm{~cm}$ and mimicked other common afflictions of oral cavity. In this case, an excision biopsy was performed and histological evidence was obtained.

Although imaging examinations suggested the presence of cancer in our patient, as we did not obtain any histological confirmation, it is difficult to affirm that a new pharyngeal tumor was the responsible for the current case report. The multifocal manifestation of aerodigestive cancers under an intact mucosa is not much explored in the literature. Nevertheless, recurrences and new tumors with this pattern of presentation have already been published and represent true diagnostic dilemmas, especially when there is no availability of diagnostic support tools, such as PET-CT ${ }^{12,13}$.

As the survival rate for head and neck cancer increases, the long-term complications of radiotherapy become more evident. A recent meta-analysis explored the long-term vascular side effects of radiotherapy on neck tissues ${ }^{14}$. The mechanisms of injury remain unclear, although most studies report only arterial damage, with carotid stenosis and progressive atherosclerosis. Our greatest limitation is that the diagnosis of tongue gangrene was exclusively based on physical examination and findings of CT. A histopathological specimen and/or vascular imaging studies, such as carotid angiography, ultrasound Doppler, or angio-CT, would probably help with etiologic diagnosis.

Even though not completely elucidated, our patient followed the dramatic evolution of those with advanced and unresectable head and neck cancers, and death occurred due to uncontrolled respiratory infection in an already debilitated individual.

\section{REFERENCES}

1. Souvirón Encabo R, García de Pedro F, Encinas A, Rodríguez A, Scola Yurrita B. Necrosis of the tongue secondary to bilateral carotid thrombosis after radiotherapy. Acta Otorrinolaringol Esp. 2007;58:331-2.

2. Kamatani T, Yamashita K, Okabayash T, Maeda H, Toi M, Yamamoto T. Bilateral ischemic necrosis of the tongue due to disseminated intravascular coagulation. Int J Oral Maxillofac Surg. 2008;37:777-9.

3. Arnung K, Nielsen IL. Temporal arteritis and gangrene of the tongue. Acta Med Scand. 1979;206:239-40.

4. Brearley BF, Macdonald JG. Temporal arteritis resulting in infected gangrene of tongue. BMJ. 1961;1:1151-2.
5. Ciurtin C, Shirodaria A, Manson J, MacMillan R. Polyarteritis nodosa associated with necrosis of the tongue: a rare presentation in an adult patient. Br J Oral Maxillofac Surg. 2015;53:883-5.

6. Korn S, Huppert A, Spitzer S, DeHoratius RJ. Systemic lupus erythematosus presenting with lingual infarction. $J$ Rheumatol. 1988;15:1281-3.

7. Storm TL, Jorgensen F. Lingual infarction and sudden blindness due to giant cell arteritis. Acta Med Scand. 1983;214:85-6.

8. Lekovic JP, Friedman CM, Desancho MT. Lingual thrombosis in a woman with antiphospholipid syndrome. Am J Obstet Gynecol. 2013;208:e3-4.
9. Buch RS, Schmidt M, Reichert TE. Acute tongue necrosis provoked by epirubicin-cyclophosphamide treatment for invasive ductal breast cancer. Mund Kiefer Gesichtschir. 2003;7:175-9.

10. Morris LG, Komisar A, Liberatore LA. Total gangrene of the oral tongue following intra-aortic balloon pump for cardiogenic shock. Otolaryngol Head Neck Surg. 2007;137:358-9.

11. Tjioe KC, Oliveira DT, Santos PS. Tongue phlebothrombosis: Pathogenesis and potential risks. Quintessence Int. 2015;46:545-8.

12. Zbären $P$, Weidner $S$, Thoeny HC. Laryngeal and hypopharyngeal carcinomas after (chemo) radiotherapy: a diagnostic dilemma. 
Curr Opin Otolaryngol Head Neck Surg. 2008;16:147-53.

13. Mancuso AA, Hanafee WN. Elusive head and neck carcinomas beneath intact mucosa. Laryngoscope. 1983;93:133-9.

14. Bashar K, Healy D, Clarke-Moloney M, Burke P, Kavanagh E, Walsh SR.
Effects of neck radiation therapy on extra-cranial carotid arteries atherosclerosis disease prevalence: systematic review and a metaanalysis. PLoS One. 2014;9:e110389.

Received: July 09, 2016

Accepted: Aug 19, 2016 